\title{
Portfólio Reflexivo Eletrônico: Resultados de um Projeto Piloto
}

\author{
Reflective e-Portfolio: Results of a Pilot \\ Project
}

\author{
Marcos Fortel \\ Wanderley Lopes de Souza $a^{I, I I}$ \\ Roseli Ferreira da Silva ${ }^{\text {Il }}$ \\ Antonio Francisco do Prado II
}

\section{PALAVRAS-CHAVE}

- Prática Profissional;

- Educação Médica;

- Aprendizagem Baseada em Problemas;

- Informática Médica.

\section{KEYWORDS}

- Professional Practice;

- Education, Medical;

- Problem-Based Learning;

- Medical Information Technology.
Recebido em: 17/04/2014

Reencaminhado em: 17/12/2015

Aprovado em: 05/04/2016

REVISTA BRASILEIRA DE EDUCAÇÃO MÉDICA

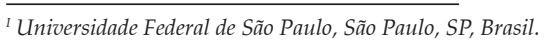

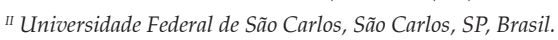

\section{RESUMO}

Este estudo investigou a implantação de um Portfólio Reflexivo Eletrônico (PRE) nas atividades de Prática Profissional (PP), que ocorrem em ambientes internos e externos ao campus. Para avaliação, um protótipo foi instalado em netbooks e usado durante cinco meses por um grupo de estudantes e um facilitador do primeiro ciclo da Unidade Educacional de Prática Profissional (Uepp) do curso de Medicina da Universidade Federal de São Carlos (UFSCar). O estudo percorreu as seguintes etapas: levantamento dos requisitos, design e implementação do protótipo e avaliação do protótipo. Na avaliação foi feito um inquérito com aplicação de dois questionários e a realização de observaçães locais durante esse período. Os resultados demonstraram que a maioria dos estudantes prefere usar o PRE nas atividades internas e em grupo, mas prefere usar papel durante o atendimento aos pacientes, $e$ que características de hardware e software do dispositivo de acesso e os diferentes ambientes de uso influenciam essa preferência.

This study investigated the deployment of a Reflective e-Portfolio (REP) for Professional Practice (PP) activities, which activities occur on and off the campus. For the prototype evaluation, the e-Portfolio was installed on netbooks and used for five months by a group of students and a facilitator of the first cycle of the Educational Unit of Professional Practice (EUPP) of the UFSCar Medicine Course. The study involved the following steps: survey to establish the requirements, design and implementation of the prototype, and evaluation of the prototype. The evaluation consisted of an investigation with the application of two questionnaires and local observations conducted during that period. The results showed that most students prefer to use the REP in the internal and group activities, but prefer to use paper for patient care, and that hardware and software characteristics of the access device as well as the different environments of use influence this preference. 


\section{INTRODUÇÃO}

O Portfólio Reflexivo (PR) é uma das ferramentas usadas no acompanhamento do processo de ensino-aprendizagem. Segundo Zubizarreta ${ }^{1}$, o PR é um instrumento flexível e baseado em produções dos estudantes, que evidencia a construção do conhecimento ao longo da disciplina e que os motiva a uma contínua reflexão e análise colaborativa de aprendizagem. Segundo Topp e Goeman², o uso consistente do PR em todas as atividades de um curso apresenta impacto positivo na aprendizagem de conteúdos.

O uso do PR tem sido descrito como uma estratégia importante em diferentes ambientes de aprendizagem ${ }^{3,4}$, bem como em ambiente de trabalho, com a finalidade de apoiar e melhorar a aprendizagem e aproximar trabalhadores da produção de conhecimento ${ }^{5}$.

O grande desenvolvimento da computação móvel ocorrido nos últimos anos, com o advento dos netbooks, smartphones e tablets, e das redes de dados sem fio, como 4G, promoveu um novo patamar de mobilidade aos usuários. Possibilitou o acesso e o processamento de informações a qualquer momento e de qualquer lugar, e forneceu o suporte computacional necessário à ligação entre as diferentes dimensões do processo de aprendizagem, sem causar interrupções ou distrações nesse processo ${ }^{6,7,8}$.

Recebe-se cada vez mais na universidade uma geração de estudantes conectados aos dispositivos móveis. Quase a totalidade dos estudantes no curso em estudo possui netbooks, smartphones ou tablets. Isto tem mudado a forma como os alunos têm estudado e armazenado seus textos, sínteses e reflexões produzidos nas unidades educacionais. Não é mais possível exigir que os estudantes acumulem textos em papel e façam cópias de todas as suas produções, que são realizadas diariamente, para colocar no portfólio de papel. No contexto do ensino da Medicina, o PR pode ser empregado tanto nas atividades simuladas como na prática profissional, possibilitando reflexões acerca do cuidado aos pacientes ${ }^{9}$.

Desta forma, houve o desenvolvimento de um Portfólio Reflexivo Eletrônico (PRE). Assim, este estudo apresenta as experiências obtidas durante a implantação de um PRE na Unidade Educacional de Prática Profissional (Uepp) do curso de Medicina da Universidade Federal de São Carlos (UFSCar). O PRE tem por objetivo substituir o portfólio atual, em papel, utilizado na Uepp do curso.

\section{AMBIENTE DO ESTUDO}

O estudo foi realizado na Uepp do primeiro ano do curso de Medicina da UFSCar. Neste curso, a proposta pedagógica é voltada para uma formação orientada por competência, segundo uma abordagem educacional socioconstrutivista, e emprega metodologias ativas de aprendizagem, tais como Problem Based Learning ${ }^{10}$ (PBL) e Practice Based Learning ${ }^{11}$.

Conforme a UFSCar ${ }^{12}$, a estrutura curricular desse curso é baseada em unidades educacionais interdisciplinares e está dividida em três ciclos de dois anos cada, onde ocorrem atividades organizadas em torno de três unidades educacionais: Unidade Educacional de Simulação da Prática Profissional (Uespp), com as atividades de situação-problema e estações de simulação; Unidade Educacional de Prática Profissional (Uepp) e Unidade Educacional Eletiva (UEE).

A Uepp, foco deste estudo, trabalha com duas estratégias educacionais, a reflexão da prática $(\mathrm{RP})$ e a atividade de prática profissional (PP). No primeiro ciclo da Uepp (primeira e segunda séries), as atividades de PP têm como cenário a área de abrangência das Unidades de Saúde da Família (USF). Além da própria USF, essa área engloba todos os domicílios e equipamentos sociais circunscritos ao território geográfico da USF, por meio dos quais são desenvolvidas ações de produção de saúde.

Na atividade de PP, cada estudante acompanha, a partir de seu primeiro semestre, uma família da área de abrangência e assim vão se somando progressivamente famílias a sua prática, totalizando dez até o final da segunda série. Esta atividade é supervisionada diretamente por um preceptor, que é um médico vinculado à USF. A ação prioritária dos estudantes na PP é o cuidado às famílias e pacientes sob sua responsabilidade, realizado por meio de visitas domiciliares, nas quais eles constroem narrativas, histórias de vida das famílias, histórias clínicas e planos terapêuticos de um paciente de cada família. Esses produtos são colocados no PR e evidenciam o processo de construção da aprendizagem do estudante.

$\mathrm{Na} R \mathrm{P}$, a atividade é realizada em grupos de oito estudantes e dispõe de um facilitador, docente do Departamento de Medicina (DMed), um cofacilitador e o preceptor, um médico da USF, na qual os estudantes desenvolvem as atividades de PP.

$\mathrm{O}$ processo de ensino-aprendizagem da RP é composto das seguintes etapas ${ }^{13}$ :

- 1ํㅡㄹ momento: síntese provisória, na qual as narrativas, histórias de vida das famílias, histórias clínicas e planos terapêuticos servem como disparadores para as atividades, considerando as capacidades prévias dos estudantes, permitindo identificar lacunas no conhecimento, levantar hipóteses e, a partir destas, construir as Questões de Aprendizagem (QA);

- $2^{\circ}$ momento: síntese individual, na qual cada estudante se responsabiliza pela busca e identificação de informações que permitam elaborar essa síntese coerentemente com as QA; - 3ํㅡㄹ momento: nova síntese, na qual os estudantes discutem os achados em grupo, com aprofundamentos conceituais, 
científicos e metodológicos, com base nas pesquisas individuais e na estruturação das QA. Toda a produção do estudante de cada um destes momentos da RP é colocada no PR.

O PR é acompanhado e avaliado (avaliação formativa) tanto pelo facilitador da RP como pelo preceptor da PP ao longo de cada série. O PR também é utilizado como estratégia pedagógica no segundo ciclo do curso (terceira e quarta séries).

\section{Portfólio Reflexivo Eletrônico (PRE)}

Usado desde a criação do curso de Medicina da UFSCar, o PR, na versão em papel, procura facilitar o processo de construção de conhecimento contextualizado, favorecendo a compreensão dos significados possíveis e a atribuição de sentidos às situações e aos conceitos que constituem o cerne da aprendizagem, estimulando o desenvolvimento crítico e reflexivo do estudante $^{14}$.

Na versão eletrônica, o PR apresenta as seguintes vantagens: é mais fácil reorganizar, editar e combinar conteúdos no PRE, sendo possível realizar buscas e acessar conteúdos de forma não linear; o estudante pode usar hyperlinks para conectar diferentes conteúdos, incluindo conteúdos de sua autoria e referências externas, o que facilita a criação de associações entre diferentes áreas do conhecimento ${ }^{15}$; por ser "portável e móvel", o PRE permite que seu conteúdo seja transportado e transferido com facilidade e acessado de locais diferentes, podendo ser compartilhado com outras pessoas, principalmente com o facilitador e o preceptor ${ }^{16}$.

\section{MATERIAIS E MÉTODOS}

O desenvolvimento e a avaliação do PRE utilizado nas atividades da Uepp foram divididos nas seguintes etapas: levantamento dos requisitos, design e implementação do protótipo, e avaliação do protótipo. Além do pesquisador principal, colaboraram com este estudo os docentes do Departamento de Medicina da UFSCar (Dmed), preceptores das USF, que são os médicos da Secretaria Municipal de Saúde (SMS), e estudantes do primeiro e segundo anos do curso de Medicina da UFSCar.

No desenvolvimento deste estudo foram salvaguardados os preceitos éticos relativos aos procedimentos de autorização institucional e parecer do Comitê de Ética em Pesquisa (CEP) da Universidade Federal de São Paulo (Unifesp). Foi também observada a resolução número 196/1996 do Conselho Nacional de Saúde, vigente no período de coleta de dados, sobre pesquisa envolvendo seres humanos, no que se refere ao termo de responsabilidade do pesquisador e de consentimento livre e esclarecido dos sujeitos que manifestaram sua anuência em participar do estudo.

\section{Coleta de dados e participantes}

A coleta de dados ocorreu em três fases: Especificação de Requisitos, Avaliação do Protótipo I e Avaliação do Protótipo II. Para a realização dessas fases foram selecionados os seguintes participantes: grupo de estudantes e facilitadores do primeiro ciclo da Uepp.

Na primeira fase, Especificação de Requisitos, fez-se um levantamento das características das atividades relacionadas à Uepp. As informações coletadas nessa fase foram essenciais para a especificação dos requisitos do protótipo do Portfólio Reflexivo Eletrônico e para a escolha do dispositivo de acesso utilizado no período de avaliação. Detalhes sobre essa fase, sobre a arquitetura e a implementação do PRE são descritos em outro artigo ${ }^{17}$.

Para as outras duas fases, relacionadas com a Avaliação dos Protótipos I e II, foi selecionado um grupo piloto, respeitando-se a seguinte regra de inclusão: todos os estudantes de um grupo de RP $(n=8)$ e o facilitador $(n=1)$ desse grupo participaram voluntariamente da avaliação, com o objetivo de evitar que parte do grupo trabalhasse com papel e o restante com o PRE, comprometendo o uso da função de compartilhamento do PRE durante as atividades do grupo de RP.

Assim, foram disponibilizados nove netbooks ASUS 701, que ficaram de posse dos estudantes e do facilitador durante todo o período da avaliação e que foram empregados nas atividades internas e externas à UFSCar. Para evitar que o uso de dispositivos de acesso com diferentes características pudesse afetar os resultados da avaliação, o PRE só foi instalado nesses netbooks. Também foram realizadas duas reuniões com o grupo piloto para treinamento no uso do PRE.

Na Avaliação do Protótipo I do PRE, realizada após três meses de uso, foi aplicado um questionário estruturado baseado no Technology Acceptance Model (TAM) ${ }^{18}$. Esse modelo parte do pressuposto de que uma intenção comportamental do indivíduo, para usar um sistema, é determinada por duas crenças: facilidade de uso percebida e utilidade percebida. Ambas medem os efeitos de variáveis externas, tais como as características do sistema e o processo de desenvolvimento na intenção de uso do sistema.

Para esta fase foi elaborado um questionário dividido em duas partes. A primeira continha três questões: sexo do usuário; se este possui computador pessoal (desktop ou notebook); e de que tipo de acesso à internet dispõe em sua residência (banda larga ou discada). A segunda parte foi organizada em quatro grupos: Grupo I, com questões genéricas sobre o uso de computador; Grupo II, com questões relacionadas ao uso do dispositivo; Grupo III, com questões relativas à facilidade de uso percebida, adaptadas de Davis ${ }^{18,19}$; Grupo IV, 
com questões relativas à utilidade percebida, adaptadas de Davis $^{18,19}$.

Nas respostas a cada questão foi utilizada uma escala de Likert de sete pontos, como sugerido por Ajzen e Fishbein ${ }^{20}$, variando gradualmente de um (discordo totalmente) a sete (concordo totalmente), com o ponto central quatro correspondendo a não discordo nem concordo. Também foi observado o uso do protótipo durante duas reuniões de RP.

Na Avaliação do Protótipo II, realizada após cinco meses de uso, foi elaborado um segundo questionário com três questões fechadas e oito questões semiestruturadas, dando liberdade ao estudante de expressar suas percepções sem as restrições de um questionário fechado. Como nessa etapa também foi avaliado o uso do PRE durante os atendimentos aos pacientes, o facilitador optou por não participar, alegando que não atuava nessa atividade.

A primeira parte desse questionário, com três questões, teve o objetivo de avaliar o uso de cada funcionalidade do PRE em atividades que ocorrem em três ambientes diferentes de aprendizagem: Q1, nas reuniões de RP, em ambiente protegido e com rede de dados; Q2, na USF e nas residências dos pacientes, em ambiente externo e não protegido e com cobertura limitada de rede de dados; Q3, nas residências dos estudantes, em ambiente externo e com rede de dados. Na análise das respostas foram usadas medidas de frequência simples.

A segunda parte do questionário (Q4 - Q11) foi composta de questões abertas sobre a experiência com o PRE e com o dispositivo de acesso. A elaboração dessas questões contou com a colaboração de dois professores do curso de Medicina. Na análise das respostas foi empregada uma metodologia qualitativa baseada na análise de conteúdo proposta por Bar$\operatorname{din}^{21}$, sendo esta análise realizada em três fases. A primeira consistiu na organização do material a ser analisado, de modo a facilitar a operacionalização e a sistematização das ideias iniciais. A segunda baseou-se na exploração do material, na qual foi feita a definição das unidades de registro (UR) e das unidades de contexto (UC). E a terceira fase consistiu no tratamento dos resultados, na inferência e na interpretação.

\section{RESULTADOS}

\section{Avaliação do Protótipo I}

Os resultados da primeira parte do questionário da Avaliação do Protótipo I demonstraram que o grupo que participou das avaliações do protótipo do PRE era composto por seis mulheres $(66,6 \%)$, incluindo a facilitadora, e três homens $(33,3 \%)$. Todos os participantes possuíam computador pessoal, sendo sete notebooks (77,7\%) e dois desktops (22,2\%), e também tinham acesso à internet através de banda larga em suas residências.
Os resultados da segunda parte desse questionário são apresentados na Tabela 1 .

\section{TABELA 1}

Medidas estatísticas das respostas da Avaliação do Protótipo I, segunda parte do questionário

Questões $\quad$ Média $^{1}$ DP

Grupo I - questões genéricas sobre o uso de computador

1 - Tenho facilidade em utilizar meu computador pessoal 5,33 1,73

2 - Tenho facilidade em navegar pela WEB utilizando meu computador

$6,00 \quad 0,50$

Grupo II - questões relacionadas ao uso do dispositivo

3 -É fácil transportar o netbook durante minhas atividades de PP

4 - Consigo digitar facilmente utilizando o teclado do netbook

5 - Apesar do tamanho reduzido da tela consigo ler os textos e páginas Web

6 - Considero a interface do netbook simples e de fácil de utilização

Grupo III - questões relativas à facilidade de uso percebida ${ }^{2}$

7 - Aprender a operar o PRE é fácil para mim 5,00

8 - Eu acho fácil de utilizar o PRE para fazer o que eu quero que ele faça

9 - Minha interação com o PRE é clara e compreensível

10 - É fácil para mim me tornar hábil em usar o PRE

11 - Considero que o PRE é fácil de usar

5,11

Grupo IV - questões relativas à utilidade percebida ${ }^{2}$

12 - Utilizar o PRE me permite realizar tarefas mais rapidamente

13 - Utilizar o PRE melhora o meu desempenho nas atividades da UEPP

14 - Utilizar o PRE aumenta minha produtividade

15 - Utilizar o PRE aumenta a minha eficácia nas atividades da UEPP

16 - Utilizar o PRE torna mais fácil a execução das atividades na UEPP

17 - Considero que o PRE é útil em minhas atividades na UEPP

(1) O maior valor é 7 (concordo totalmente) e o menor é 1 (discordo totalmente). (2) Grupo de questões adaptadas de Davis ${ }^{15,16}$.

\section{Avaliação do Protótipo II}

Os resultados da primeira parte do questionário da Avaliação do Protótipo II (Figura 1) indicaram que o emprego do PRE se concentrou em dois ambientes de aprendizagem: durante 


\section{Gráfico de funcionalidade segundo o ambiente de aprendizagem}

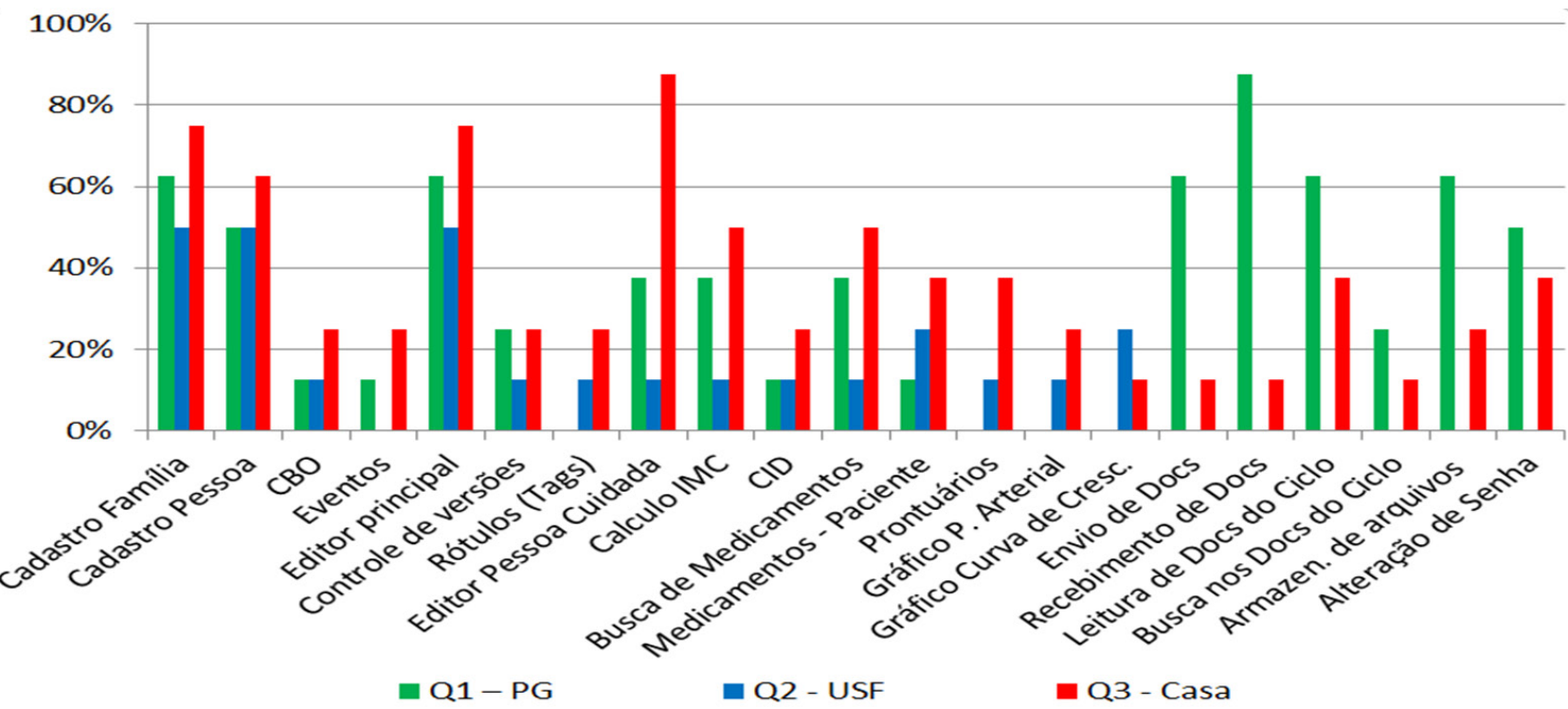

as atividades de RP, para compartilhamento de informações; e nas residências dos estudantes, para digitação das informações coletadas durante as visitas aos pacientes e elaboração das sínteses e narrativas.

O Quadro 1 apresenta um sumário da análise de conteúdo das questões Q4 a Q11 da Avaliação do Protótipo II. Cada questão foi analisada individualmente e, quando possível, foram extraídas unidades de contexto. Por exemplo, 62,5\% das respostas da $\mathrm{Q} 4$ tinham relação com o contexto de entrada e saída de dados do netbook, e $40 \%$ das respostas relativas a esse contexto consideraram que o tamanho do teclado era bom.

\section{QuAdro 1}

\section{Sumário da análise de conteúdo das questões abertas (Q4 - Q11) da Avaliação do Protótipo II}

\section{Questão}

Unidades de Contexto

Entrada e visualização de dados no netbook $(62,5 \%)$

Q4. Descreva a sua experiência com o hardware do netbook (ex.: tela, teclado, etc.), incluindo o sistema operacional instalado (Linux).

\section{Unidades de Registro}

\begin{tabular}{|l|}
\hline Tamanho do teclado adequado $(40 \%)$ \\
\hline Tamanho do teclado pequeno $(40 \%)$ \\
\hline Tamanho da tela adequado $(40 \%)$ \\
\hline Tamanho da tela pequeno $(60 \%)$ \\
\hline Letra pequena (20\%) \\
\hline Dificuldade no uso $(100 \%)$ \\
\hline Prefere Windows $(50 \%)$ \\
\hline Não se adaptou $(33 \%)$ \\
\hline Adaptação requer tempo $(66 \%)$ \\
\hline Peso extra incomoda $(50 \%)$ \\
\hline Fácil de transportar $(50 \%)$ \\
\hline
\end{tabular}


QuAdro 1

\section{Sumário da análise de conteúdo das questões abertas (Q4 - Q11) da Avaliação do Protótipo II}

Questão

Q5. Além do PRE, quais aplicativos disponíveis no netbook você utilizou?

Q6. Você tem algum receio em utilizar o netbook durante o atendimento aos pacientes? Comente sua resposta.

Q7. Que alterações você sugere ao PRE?

Q8. Avalie sua experiência no uso do PRE, destacando os fatos positivos e negativos.

Q9. No próximo semestre, você prefere trabalhar com a versão eletrônica ou retornar à versão em papel do portfólio? Comente sua resposta.

Q10. Que ferramentas da internet você utiliza em suas atividades acadêmicas, incluindo a troca de informações entre os componentes do pequeno grupo (e-mail, Google Docs, etc.)?

Q11. Você tem alguma observação e/ou sugestão adicional a fazer?
Unidades de Contexto

Unidades de Registro

Navegador Web (100\%)

Pacote Office (Open Office) $(50 \%)$

$\begin{array}{ll}\text { Aplicativos utilizados além do } & \text { Telefonia IP (Skype) (25\%) }\end{array}$

PRE $(100 \%)$

Multimídia Player (SMPlayer) (25\%)

Leitor PDF (Acrobat) (12,5\%)

Ger. de Arquivos (Xandros-explorer) (12,5\%)

$\operatorname{Sim}(75 \%)$

Não $(25 \%)$

Digitação diminui a atenção ao paciente (75\%)

Afeta o relacionamento

estudante-paciente $(50 \%)$

O paciente não se sente confortável (50\%)

Confiabilidade (25\%)

Medo de ter o netbook roubado (50\%)

Medo de perda de dados (50\%)

Adicionar um módulo de relatórios (33,3\%)

Gerais $(37,5 \%)$

Alterar alguns ícones (33,3\%)

Maior velocidade de execução (33,3\%)

Log da última atualização do paciente $(33,3 \%)$

Controle do tamanho de letra (40\%)

Revisor ortográfico (20\%)

Editores de texto $(62,5 \%)$

Melhor identificação dos editores (20\%)

Editor de rascunho (20\%)

Melhor organização das informações (50\%)

Fatos positivos

$(100 \%)$

Facilidade na troca de informações no grupo $(37,5 \%)$

Ferramentas de suporte (ex.: busca CID) (12,5\%)

Dificuldades na operação do PRE (25\%)

Falhas na infraestrutura de rede sem fio (25\%)

Diminui a atenção ao paciente $(12,5 \%)$

Fatos negativos

$(100 \%)$

Treinamento insuficiente $(12,5 \%)$

Dúvidas sobre a confiabilidade do armazenamento das informações (12,5\%)

Mudanças dos pacientes e de USF durante a avaliação (12,5\%)

Prefere o portfólio em papel $(62,5 \%)$

Durante o atendimento ao paciente $(100 \%)$

Prefere o portfólio eletrônico $(12,5 \%)$

Prefere ambos $(12,5 \%)$

Indeciso (12,5\%)

Prefere o portfólio em papel $(12,5 \%)$

\begin{tabular}{l|l} 
Durante as reuniões de & Prefere o portfólio eletrônico $(62,5 \%)$
\end{tabular}

\begin{tabular}{l|l} 
pequeno grupo $(100 \%)$ & Prefere ambos $(12,5 \%)$
\end{tabular}

Indeciso $(12,5 \%)$

E-mail $(100 \%)$

Busca Web (100\%)

\begin{tabular}{l|l} 
Ferramentas de internet & Google Docs $(37,5 \%)$
\end{tabular}

utilizadas $(100 \%)$

Bases médicas $(25,0 \%)$

Redes sociais $(12,5 \%)$

Mensagens instantâneas (12,5\%)

Netbook com um teclado maior e MS Windows (12,5\%)

Mais treinamento $(12,5 \%)$

Deseja saber o resultado das avaliações $(12,5 \%)$
Observações/sugestões adicionais $(37,5 \%)$ 


\section{ANÁLISE DOS RESULTADOS E DISCUSSÃO}

A análise e a discussão dos resultados foram organizadas de modo a destacar os principais aspectos da implantação do PRE.

\section{Arquitetura e implementação do PRE}

A avaliação validou a arquitetura e as tecnologias empregadas na implementação do $\mathrm{PRE}^{17}$, já que possibilitou aos usuários utilizar o protótipo em todos os ambientes de aprendizado da Uepp, incluindo os ambientes externos desprovidos de redes de dados. Além disso, não houve nenhum comentário dos usuários quanto ao não funcionamento do PRE durante as atividades realizadas.

Também foram efetuados testes em laboratório com outros tipos de dispositivo de acesso (ex.: smartphones, tablets) para avaliar a capacidade de adaptação da interface do PRE a diferentes dispositivos. Embora tenha sido observada a necessidade de algumas melhorias na interface, o PRE funcionou adequadamente.

Várias modificações de interface e a adição de novas funcionalidades foram sugeridas pelos usuários (Q7 - Quadro 1). Essas sugestões serão incorporadas nas próximas versões do PRE, sem a necessidade de alterar as tecnologias empregadas no desenvolvimento do protótipo ${ }^{17}$.

\section{Experiência anterior no uso do Portfólio Reflexivo}

Neste grupo estudado, os estudantes já utilizavam o Portfólio Reflexivo desde o ingresso no curso de Medicina, mas tinham pouca experiência em usá-lo nas atividades de atendimento aos pacientes. Um dos motivos dessa pouca experiência foi a demora na definição da USF, devido a mudança e reformas na unidade, ocasionando um atraso na definição das famílias atendidas. Esse fato fez com que os estudantes vivenciassem duas novas experiências simultaneamente: a aproximação dos pacientes e o uso do PRE.

Cinco estudantes já utilizavam seus notebooks, mas apenas durante as reuniões de RP, na universidade. Embora esta contasse com edificações recém-construídas, não dispunha de uma infraestrutura adequada de tomadas elétricas que suportasse o uso de notebooks por todos os estudantes e também não havia cobertura de rede sem fio. Dessa forma, os estudantes tinham dificuldade em realizar pesquisas devido à falta de acesso à internet. Basicamente, utilizavam o aplicativo MS Word e trocavam informações via pen drive.

\section{Percepções dos usuários sobre o uso do Portfólio Eletrônico}

Segundo as respostas da Avaliação do Protótipo I, os usuários consideraram que o PRE é de fácil uso e útil para a realização das atividades da Uepp. É importante citar que, até a aplica- ção desse questionário, os estudantes não haviam utilizado o PRE nas atividades de atendimento aos pacientes, na PP, devido a problemas operacionais da USF.

Na Avaliação do Protótipo II, os estudantes destacaram como aspectos positivos o auxílio na organização dos dados coletados e a facilidade no compartilhamento eletrônico de informações entre os integrantes do grupo de RP. Desta forma, foi eliminada a necessidade de cópias reprográficas dos textos estudados e elaborados para as reuniões de RP.

Quanto à dificuldade de utilização, citada como aspecto negativo, na experiência durante as observações e no suporte fornecido aos usuários, ficou demonstrado que a maioria das dificuldades consistia em como se conectar à rede, configurar a impressora e acessar o pen drive, ou seja, relacionadas ao sistema operacional do dispositivo de acesso. Com mais treinamento e a presença de uma equipe de suporte técnico de informática da UFSCar, é possível diminuir essas dificuldades.

Uma possibilidade de aprimoramento do suporte, descrita em Belcher et al. ${ }^{22}$, seria definir um estudante "tutor", em cada grupo de RP, que receberia treinamento adicional sobre o uso do PRE e acesso direto à equipe de suporte para consultas mais complexas. Os outros estudantes seriam incentivados a esclarecer suas dúvidas primeiramente com o estudante "tutor".

Alguns estudantes demonstraram preocupação com a segurança do PRE, como o receio quanto ao roubo do equipamento e à perda das informações digitadas. Green et al..$^{23}$ também descrevem a preocupação em questões relacionadas à possibilidade de roubo de dados e à perda de documentos importantes devido a falha de software. Acredita-se que esse receio deva se dissipar com o tempo, pois durante todo o período de avaliação não houve nenhuma ocorrência de roubo ou extravio dos netbooks; quanto à perda de dados, as ocorrências verificadas foram relativas a erros de uso, sendo que os dados perdidos foram recuperados da cópia de segurança.

Outro receio dos estudantes foi o controle de acesso às informações armazenadas no PRE. No portfólio baseado em papel, caso desejassem remover alguma informação, bastava retirar uma folha da pasta. Esse receio foi tratado na fase de implementação do PRE, possibilitando ao usuário definir a permissão de acesso (apenas o criador; todos; facilitador; preceptor; facilitador e preceptor) para cada documento gerado, sendo que o padrão é que apenas o criador do documento tenha acesso.

\section{Percepção do facilitador do grupo de RP}

Durante os cinco meses de avaliação do protótipo, o facilitador utilizou o PRE nas reuniões de grupo. Apesar de descrever dificuldades relacionadas à infraestrutura e ao sistema opera- 
cional utilizado no netbook, o facilitador apreciou a facilidade de acesso aos textos dos estudantes e a redução do volume de papel propiciado pelo uso do PRE. Esses resultados foram semelhantes aos obtidos por Driessen et al. ${ }^{9} \mathrm{em}$ seu estudo, no qual o facilitador considerou mais fácil e rápida a busca e a leitura dos textos no computador do que no papel.

\section{Percepções dos usuários quanto ao dispositivo de acesso utilizado}

Como na fase de levantamento de requisitos não foi localizado nenhum estudo que avaliasse o uso de netbooks em portfólios eletrônicos, foram incluídas nas avaliações do PRE várias questões relativas a esse tipo de dispositivo.

Nas questões relacionadas ao dispositivo de acesso (Grupo II - Tabela 1), as referentes ao teclado e à tela do netbook foram as que obtiveram as médias mais baixas. Como o teclado do netbook utilizado corresponde a $82 \%$ do tamanho do teclado dos notebooks e desktops, e como o volume de texto digitado é significativo, acredita-se que os usuários tiveram pouco tempo para se adaptar às características do dispositivo. Cabe salientar ainda que os próprios estudantes consideram o tamanho e o peso de seus notebooks como fatores impeditivos ao uso destes em atividades externas.

Mesmo usando um dispositivo com maior capacidade de entrada e saída de dados que os Personal Digital Assistants (PDAs) e smartphones, as avaliações revelaram ser difícil conciliar mobilidade com facilidade na entrada e visualização das informações coletadas, dificuldade acentuada pelas características das informações registradas em um portfólio. Os resultados obtidos indicaram que o teclado reduzido do netbook desestimulou o uso desse dispositivo durante as atividades de atendimento ao paciente. Garret e Jackson ${ }^{4}$ também observaram que os estudantes preferiam digitar em casa, usando seus desktops e notebooks, as informações coletadas em ambientes externos.

Cotterill et al. ${ }^{24}$ verificaram que, durante as atividades de habilidades clínicas, havia uma preferência pelo portfólio de papel, sobretudo por parte dos estudantes com pouca experiência no uso de computadores. Horner ${ }^{25}$ observou que, apesar da percepção de vantagens do portfólio eletrônico, a maioria dos estudantes preferiu continuar com os portfólios de papel. Ainda de acordo com esse trabalho, a principal razão para o ocorrido foi a curta duração dos módulos do curso, que impediu a introdução gradativa do portfólio eletrônico e não outorgou o tempo necessário à adaptação dos estudantes a ele.

Embora a arquitetura desenvolvida para o PRE possibilite o uso de smartphones, as restrições de interface de entrada/ saída de dados desses dispositivos impedem seu pleno em- prego nas atividades do curso. Mesmo assim, tais dispositivos podem aumentar o grau de ubiquidade do PRE já que podem ser empregados quando a quantidade de dados a ser introduzida é pouca, tal como numa consulta às informações de um paciente e/ou na realização de pequenos registros de um atendimento.

Em relação ao Sistema Operacional (SO) dos netbooks utilizados - uma versão customizada do Linux -, os respondentes alegaram falta de experiência com ele e dificuldades em realizar determinadas operações, como impressão e configuração do acesso à rede, incluindo a impossibilidade de executar alguns softwares normalmente disponíveis no ambiente Windows (ex.: MS Office). Como o PRE é uma aplicação web, os usuários não sentiram dificuldade no uso, pois o navegador e o PRE têm a mesma interface e funcionalidade, seja qual for o SO, Linux ou Windows. Mesmo assim, o SO foi uma barreira importante na adaptação dos usuários ao uso dos netbooks.

O hábito com as aplicações do ambiente Windows e a dificuldade em usar aplicações semelhantes no netbook, que dispõe de outro SO, levaram alguns estudantes a transportar dois computadores, ou seja, o próprio e o netbook. Garret e Jackson ${ }^{4}$ forneceram um $P D A$ contendo um portfólio eletrônico e 21 aplicativos, sendo que o e-portfólio ficou em 13ํํำ lugar quanto ao número de utilizações. Isto demonstra que devem ser levadas em consideração as aplicações comumente utilizadas pelos usuários na definição do dispositivo de acesso e do SO a ser utilizado.

Essas dificuldades deverão ser amenizadas quando os netbooks utilizados forem substituídos por uma nova geração, que oferece tela e teclado maiores e autonomia de bateria estendida, possibilitando seu emprego em todas as atividades do curso. Essa nova geração também já emprega o SO Windows, aos quais os estudantes estão habituados.

\section{Influência de diferentes ambientes de aprendizagem}

Os estudantes usaram o PRE em três ambientes de aprendizagem: durante as reuniões de RP, nos atendimentos aos pacientes e durante as atividades de pesquisa individual realizadas no campus ou na própria residência.

O uso do PRE durante as reuniões de grupo de RP foi o que mais agradou aos usuários, principalmente em relação à facilidade de compartilhar textos entre os integrantes do grupo. Esse compartilhamento também auxiliou no acompanhamento e na avaliação dos estudantes, na medida em que os facilitadores e preceptores conseguiram, a qualquer hora e de qualquer lugar, obter informações atualizadas dos portfólios de seus estudantes. No estudo com portfólios baseados na web, relatado por Driessen et al. ${ }^{9}$, os estudantes também con- 
sideraram o compartilhamento uma das vantagens desse tipo de portfólio.

Em relação ao uso do PRE durante o atendimento aos pacientes (Q6 - Quadro 1), a maioria declarou ter reservas, pois considera que seu uso afeta o relacionamento estudante-paciente. Entre os motivos alegados, o primeiro foi a dificuldade em conversar e digitar ao mesmo tempo, e o segundo foi que o uso de equipamentos tecnológicos em habitações muito humildes pode gerar uma barreira no atendimento.

Quanto ao primeiro motivo, deve-se levar em conta que os estudantes tinham pouca experiência tanto no uso do PRE quanto no atendimento aos pacientes e, portanto, o fator insegurança deve ser considerado. Nesse aspecto, Alsos et al. ${ }^{26}$ descrevem três fatores que afetam a comunicação entre o estudante/médico e o paciente: o tamanho e a forma do dispositivo de acesso; a interface de usuário da aplicação; a prática na comunicação com os pacientes.

Quanto ao segundo motivo, alguns estudantes passaram a encará-lo de outra forma, segundo descrito no fragmento da resposta do estudante E4:

\begin{abstract}
"Inicialmente, tinha o receio de intimidar a pessoa, por chegar com um objeto de alta tecnologia em um ambiente simples, no qual muitas vezes não haveria nem condições mínimas adequadas para viver. Depois, revi minha posição, porque ouvi experiências de profissionais de saúde e colegas de trabalho, no sentido de que um paciente se sente valorizado quando nos mostramos bem vestidos para encontrá-lo ou nos utilizamos de recursos dos melhores possíveis para atendê-lo. Agora considero que o paciente eventualmente pode se sentir valorizado por utilizar um netbook".
\end{abstract}

Observou-se que o editor do paciente era pouco usado durante as atividades de atendimento, sendo que os estudantes preferiam armazenar todas as informações num único texto para organizá-las posteriormente em suas residências. Esse procedimento reproduz o que já era feito com o portfólio baseado em papel, pois os estudantes utilizavam apenas um bloco de bolso onde faziam as anotações relativas ao atendimento e depois as repassavam para o portfólio. Observou-se também que as funcionalidades do PRE que exigiam menos digitação e as que continham componentes de auxílio à entrada de dados (ex.: preenchimento automático) foram as mais utilizadas nos atendimentos.

As diferenças entre os vários ambientes de aprendizagem influenciaram a decisão dos estudantes quanto à continuidade do uso do PRE ou ao retorno do uso do portfólio em papel (Q9 - Quadro 1). Categorizando-se as respostas, verificou-se que os próprios respondentes definiram dois ambientes de uso: nas reuniões de RP e durante o atendimento aos pacientes. A maioria dos respondentes deseja continuar usando o PRE (62,5\%) nas atividades em grupo, sendo que essa tendência se inverte quando é considerado o uso do PRE no atendimento aos pacientes.

\section{LIMITAÇÕES DO ESTUDO}

Este estudo possui algumas limitações e por esse motivo os resultados apresentados devem ser interpretados com cautela. O principal fator limitante foi o pequeno número de participantes na pesquisa, que ficou restrito à quantidade de netbooks disponíveis para a avaliação. Além disso, durante a avaliação ocorreram alguns problemas que influenciaram de alguma forma os resultados obtidos e que são descritos a seguir.

O curso de Medicina da UFSCar iniciou suas atividades em 2006, e a sua infraestrutura física e de pessoal ainda não estava completa. Por exemplo, não havia pessoal de suporte às Tecnologias de Informação (TI) para esclarecer as dúvidas dos estudantes e facilitadores relacionadas ao uso do netbook e do Linux.

Outro fato ocorrido durante a avaliação, e que prejudicou o uso dos netbooks nas atividades externas, foi uma mudança imprevista de USF e a consequente mudança dos pacientes. Isso demandou um novo período de aproximação entre os novos pacientes e os estudantes, inibindo temporariamente o uso dos netbooks e diminuindo o tempo de uso do PRE.

Esses problemas afetaram o tempo necessário à assimilação de uma nova tecnologia, no caso o uso do PRE e do netbook, o que geralmente influencia a satisfação do usuário. Kay $^{27}$ constatou que alunos de graduação matriculados num programa que emprega computadores móveis ficaram mais satisfeitos, menos ansiosos e menos irritados quando o seu conhecimento do uso de computadores aumentou ao longo do tempo. Portanto, é possível que o pouco tempo de aprendizado no uso de uma nova tecnologia tenha sido essencial na decisão dos alunos de retornar ao uso do portfólio em papel nas atividades de atendimento ao paciente.

\section{CONSIDERAÇÕES FINAIS}

O uso da tecnologia móvel na educação é uma iniciativa recente, mas a sua adoção apresenta um rápido crescimento devido à disponibilidade e à evolução dos dispositivos móveis ${ }^{27}$. Recentemente, vêm sendo desenvolvidas várias pesquisas e aplicações em computação móvel no âmbito da aprendizagem. É importante ressaltar que, no caso do curso de Medicina da UFSCar, este recurso está em consonância com o próprio modelo pedagógico e metodológico desenvolvido pelo curso. 
O PRE, utilizado na Uepp, provou ser funcional, com algumas limitações de interface, em todas as atividades realizadas, incluindo as de atendimento aos pacientes, que geralmente ocorrem em áreas remotas, onde a conectividade é restrita. $\mathrm{O}$ facilitador apreciou a facilidade de acesso aos textos produzidos pelos estudantes e a diminuição do volume de papel.

As avaliações revelaram que a maioria dos estudantes prefere usar o PRE em atividades de RP, principalmente devido à funcionalidade de compartilhamento de informações. Entretanto, estão relutantes em usar o PRE durante o atendimento aos pacientes, temerosos de se distrair com os dispositivos móveis e, desta forma, interferir na comunicação e interação com o paciente, preferindo continuar a registrar os dados coletados em papel.

Os dispositivos móveis podem apoiar as atividades do estudante, mas suas atuais características e limitações impedem a realização eficiente de algumas tarefas, tais como a leitura e digitação de longos textos. Portanto, questões relacionadas à interface devem ser consideradas cuidadosamente, já que influenciam diretamente a satisfação do usuário.

A experiência demonstrou a dificuldade de desenvolver uma aplicação que seja utilizada em diversos ambientes de aprendizagem, que possuem diferentes características de uso. Uma possível abordagem é o desenvolvimento de múltiplas interfaces: uma simplificada, usada nos atendimentos aos pacientes e contendo apenas as funcionalidades efetivamente utilizadas pelos estudantes nesses atendimentos; e outra mais completa, usada no campus e na casa do estudante, contendo todas as funcionalidades do PRE.

No PRE podem ser disponibilizados outros documentos e instrumentos de acordo com o cenário e contexto em que estiver sendo utilizado. Especificamente no contexto da Saúde da Família, no qual a Uepp é desenvolvida, podem ser incluídos o genograma, mapas das famílias e outros instrumentos importantes para a abordagem familiar e comunitária. Desta forma, o PRE também pode ser útil a toda a equipe de Saúde da Família.

Outro aspecto importante a considerar é a curva de aprendizado de uma nova tecnologia. Os estudantes nunca haviam trabalhado com o PRE, e o curto espaço de tempo para a familiarização com essa ferramenta, bem como os problemas ocorridos no período de avaliação podem ter dificultado a superação das dificuldades iniciais e uma avaliação mais criteriosa dos benefícios do PRE. Assim, são necessárias novas avaliações sobre a percepção dos benefícios e limitações do PRE, envolvendo também os professores, a fim de melhor compreender sua contribuição na prática educativa.

O PRE poderá ser utilizado em qualquer das unidades educacionais do curso de Medicina da UFSCar, além da Uepp para o qual foi desenhado, bem como em qualquer curso da área de saúde, desde que seja adequado ao propósito do curso e da unidade educacional ou disciplina em que se deseja utilizá-lo. Para esta nova utilização, será necessário um levantamento de novos requisitos de acordo com as características de cada ambiente de aprendizagem.

\section{REFERÊNCIAS}

1. Zubizarreta J. The Learning Portfolio: Reflective Practice for Improving Student Learning. San Francisco: Jossey-Bass; 2009.

2. Topp NW, Goeman RL. Perceptions of teacher candidates on eportfolio use. In: Cambridge D, Cambridge B, Yancey K. (Ed.). Electronic Portfolios 2.0. Sterling, VA: Stylus Publishing; 2009.

3. Ahmed E, Ward R, White S, Jabbar A. Use of Emerging Mobile Technologies in Portfolio Development. 2011. [capturado 13 mar. 2014]. Disponível em: http:/ / eprints.hud. ac.uk/11879/

4. Garrett BM, Jackson C. A mobile clinical e-portfolio for nursing and medical students, using wireless personal digital assistants (PDAs). Nurse Education in Practice 2006; 6(6):339-346.

5. Barrett HC. Using Mobile Devices to Develop ePortfolios. 2009-2012, updated May 8, 2013. [capturado 20 fev. 2014]. Disponível em: http:/ / electronicportfolios.org/mobile/

6. Kimber K, Wyatt Smith C. Using and creating knowledge with new technologies: a case for students as designers. Learning Media Technology 2006; 31(1):19-34.

7. Waycott J, Jones A, Scanlon E. PDAs as lifelong learning tools: an activity theory based analysis. Learning Media Technology 2005; 30(2):107-130.

8. Chertoff J. Global differences in electronic portfolio utilization - a review of the literature and research implications. Journal of Educational Evaluation for Health Professions. 2015; 12:15.

9. Driessen EW, Muijtjens AM, Van Tartwijk J, VanDer Vleuten CP. Web- or paper-based portfolios: is there a difference? Med Educ 2007; 41(11):1067-1073.

10. Rhem J. Problem Based Learning: An Introduction. The National Teaching and Learning Forum. 1998; 8(1):1-4.

11. Carlisle C, Calman L, Ibbotson T. Practice-based learning: the role of practice education facilitators in supporting mentors. Nurse Education Today 2009; 29(7):715-721.

12. UFSCAR. Caderno do Curso de Medicina. São Carlos: UFSCAR; 2008.

13. Silva RF, Francisco MA. Portfólio reflexivo: uma estratégia para a formação em medicina. Rev. Bras. Educ. Med. 2009; 33(4):562-570. 
14. Silva RF, Sa-Chaves I. Formação reflexiva: representações dos professores acerca do uso de portfólio reflexivo na formação de médicos e enfermeiros. Interface Comunicação, Saúde, Educação (Botucatu) 2008; 12(27):721-734.

15. Yancey KB. Digitized Student Portfolios. In: Cambridge B L. (Ed.). Electronic Portfolios: Emerging Practices in Student. Washington: American Association for Higher education; 2001.

16. Stefani L, Mason, R, Pegler C. The educational potential of e-portfolios: Supporting personal development and reflective learning. Connecting with elearning [on line]. 2007. [capturado 20. out 2010]. Disponível em: http://connectwithelearning.wordpress.com/2009/07/15/about-the-educational-potential-of-eportfolios-stefani-mason-and-pegler-2007.

17. Forte M, Souza WL, Silva RF, Prado AF. Portfólio Reflexivo Eletrônico na Unidade Educacional de Prática Profissional do Curso de Medicina da UFSCar. Anais do 29. Congresso da Sociedade Brasileira de Computação. X Workshop de Informática Médica; 2010, Julho. 1566-1575. Belo Horizonte, Brasil. Porto Alegre: SBC; 2010. [capturado 20. out 2010]. Disponível em: <http://www.inf.pucminas.br/ sbc2010/anais/pdf/wim/st04_03.pdf $>$.

18. Davis FD. Perceived Usefulness, Perceived Ease of Use, and User Acceptance of Information Technology. MIS Quarterly 1989; 13(3):319-340.

19. Davis FD. A technology acceptance model for empirically testing new end-user information systems: theory and results. Management Science. Massachusetts; 1986. Doutorado [Tese] - Massachusetts Institute of Technology,

20. Ajzen I, Fishbein M. Understanding attitudes and predicting social behavior. New York: Prentice-Hall; 1980.

21. Bardin L. Análise de Conteúdo. Lisboa: Edições 70; 2009

22. Belcher. Qualitative study of the impact of an authentic electronic portfolio in undergraduate medical education. BMC Medical Education 2014; 14:265.
23. Green J, Wyllie A, Jackson D. Electronic portfolio in nursing education: A review of the literature. Nurse Educ Pract. 2014;14:4-8.

24. Cotterill S, Horner P, Edney M. EPICS-2. FINAL REPORT (on line). 2009. [capturado 18 Dez, 2009]. Disponível em: <http://www.epics.ac.uk/fdtl4_docs /62_EPICS-2_final_ report_for_printing.pdf $>$.

25. Horner P. Mobile Technologies in EPICS-2 . A report produced as part of the North East regional collaboration for personalised, work -based, and life-long learning (on line). 2009. [capturado 18 Dez. 2009]; 1-17. Disponível em: <http://www.eportfolios.ac.uk/ EPICS/report/Mobile_ Technologies_in_EPICS.pdf $>$.

26. Alsos OA, Das A, Svanæs D. Mobile health IT: The effect of user interface and form factor on doctor-patient communication. Int J Med Inform. 2012;81(1):12-28.

27. Kay R. Exploring the relationship between emotions and the acquisition of computer knowledge. Computers \& Education 2008; 50(4):1269-1283.

\section{CONTRIBUIÇÃO DOS AUTORES}

Os autores trabalharam juntos nas diferentes etapas de produção do manuscrito. $\mathrm{O}$ autor principal fez a articulação das partes produzidas e a revisão final do artigo.

\section{CONFLITO DE INTERESSES}

Os autores declaram não haver conflito de interesses.

\section{ENDEREÇO PARA CORRESPONDÊNCIA}

Marcos Forte

Rua Botucatu, 862

Vila Clementino - São Paulo

CEP: 04023-062 SP

E-mail: marcos.forte@fsa.br 